\title{
RNA-Seq Reveals Underlying Transcriptomic Mechanisms of Bone Marrow-Derived Mesenchymal Stem Cells in the Regulation of Microglia-Mediated Neuroinflammation After Subarachnoid Hemorrhage
}

\author{
Lifang Zhang, ${ }^{1,2,{ }^{*}}$ Kecheng Guo,, ${ }^{1, *}$ Shigang Yin, ${ }^{3}$ Jianhua Peng, ${ }^{1}$ Jinwei Pang, ${ }^{1}$ Ning Ma, ${ }^{4}$ \\ Xianhui Zhang, ${ }^{2}$ Yuke Xie, Ligang Chen, ${ }^{1,2}$ and Yong Jiang ${ }^{1-3,5}$
}

\begin{abstract}
Subarachnoid hemorrhage (SAH) is a life-threatening cerebrovascular disease with high rates of morbidity and mortality. Microglia, the resident immune cells of the central nervous system, are involved in initiating inflammatory response post-SAH through releasing a variety of inflammatory mediators. Regulation of neuroinflammation triggered by activated microglia has become a promising therapeutic strategy for SAH. Recent studies reported that bone marrow-derived mesenchymal stem cells (BM-MSCs) have therapeutic effects, resulting from the regulation of microglia activation and production of inflammatory cytokines post-SAH. However, the underlying molecular mechanisms of BM-MSCs in targeting microglia-mediated neuroinflammation after SAH are still unclear. In this study, we used murine microglia cell line BV2 treated with oxyhemoglobin $(\mathrm{OxyHb})$ to mimic the SAH conditions in vitro. The results showed that BM-MSCs coculture modulated OxyHb-induced BV2 activation as well as polarization. We further implemented RNA-seq approaches to investigate differences in transcriptomes between OxyHb-stimulated BV2 cocultured with and without BM-MSCs. The RNA-seq results suggested that the levels of inflammatory genes were strongly altered when OxyHb-stimulated BV2 cells were cocultured with BM-MSCs. Moreover, we identified epigenetic regulators involved in the regulation of microglia-mediated inflammation by BM-MSCs. This study clarifies detailed transcriptomic mechanisms underlying the interaction between BM-MSCs and activated microglia and may lead to a new therapeutic strategy using stem cell therapy for SAH.
\end{abstract}

Keywords: mesenchymal stem cells, subarachnoid hemorrhage, microglia, neuroinflammation, epigenetic regulators

\section{Introduction}

$S^{t}$ UBARACHNOID HEMORRHAGE (SAH) is a devastating cerebrovascular disease with high mortality and morbidity rates, especially in the young stroke population [1]. Despite the advances achieved in microsurgical and endovascular surgical techniques, the outcome of SAH patients remains unsatisfactory. Recent studies have shown that early brain injury (EBI), which refers to whole brain injury within the first $72 \mathrm{~h}$ post-SAH, was one of the major causes of mortality and unfavorable outcomes in SAH patients [2,3]. As reported, multiple physiological abnormalities during EBI trigger the activation of inflammatory cascades, which result in bloodbrain barrier disruption and neuronal degeneration [4]. Therefore, it is critical to explore the mechanisms of neuroinflammation and to discover effective therapies targeting the neuroinflammatory processes in SAH [5].

Microglia, the resident immune cells of the central nervous system (CNS) [6], play a significant role in initiating neuroinflammation post-SAH. Upon sensing imbalances of environmental homeostasis, microglia are rapidly activated and polarize into two extreme states: classical (M1) and alternative (M2) [7-9]. The M1 microglia are associated with a proinflammatory cellular state that has elevated expression

\footnotetext{
${ }^{1}$ Department of Neurosurgery and ${ }^{4}$ Inflammation \& Allergic Diseases Research Unit, The Affiliated Hospital of Southwest Medical University, Luzhou, China.

${ }^{2}$ Neurosurgery Clinical Medical Research Center of Sichuan Province, Luzhou, China.

${ }^{3}$ Laboratory of Neurological Diseases and Brain Functions, Clinical Medical Research Center, The Affiliated Hospital of Southwest Medical University, Luzhou, China.

${ }^{5}$ Nuclear Medicine and Molecular Imaging Key Laboratory of Sichuan Province, Luzhou, China.

*These authors contributed equally as co-first authors.
} 
of inflammatory cytokines, including IL- $1 \beta$, TNF- $\alpha$, and IL-6, which contribute to neuronal dysfunction and cell death [10]. In contrast, the $\mathrm{M} 2$ microglia release anti-inflammatory mediators, including IL-4, IL-10, and TGF- $\beta$, leading to beneficial neuroprotection [11]. Regulation of microgliamediated inflammation has been considered an attractive approach to manage progression of EBI after SAH.

One attractive approach beginning to emerge is the use of stem cell therapy. Owing to their ability to downregulate inflammatory condition through the release of a variety of bioactive molecules, mesenchymal stem cells (MSCs) appear to be an ideal cellular source for the repair of CNS injuries [12-15]. Lately, several studies have reported the neuroprotective effects of bone marrow-derived mesenchymal stem cells (BM-MSCs) in the treatment of EBI after experimental SAH. It is shown that treatment with BMMSCs inhibited microglia activation and decreased the expression of inflammatory cytokines at 24 and $72 \mathrm{~h}$ post-SAH $[16,17]$. However, further studies are warranted to elucidate the underlying molecular mechanisms of BM-MSCs in targeting microglia-mediated neuroinflammation post-SAH.

In this study, we used the oxyhemoglobin (OxyHb)-induced BV2 microglia as an in vitro model of SAH and implemented RNA-seq approaches to investigate the differences in transcriptomes between OxyHb-stimulated BV2 cells cocultured with and without BM-MSCs. This study is fundamental for a better understanding of the biological interactions between MSCs and microglia and to progress toward clinical application of MSCs in cell therapy strategies for SAH.

\section{Materials and Methods}

\section{BM-MSCs isolation and culture}

BM-MSCs were isolated from 8-12-week-old male C57BL/ $6 \mathrm{~J}$ mice. All study procedures were approved by Institutional Animals Ethics Committees (IAEC) at Southwest Medical University, Luzhou, China (permit number: SYXK [Chuan] 2018-065), and complied with the Guidelines of the National Institutes of Health on the Care and Use of Laboratory Animals. The cells were isolated from the tibias and femurs and centrifuged at $1,000 \mathrm{rpm}$ for $10 \mathrm{~min}$ at room temperature, then washed with Dulbecco's modified Eagle's medium (DMEM; HyClone) supplemented with $10 \%$ fetal bovine serum (FBS; Gibco), $100 \mathrm{U} / \mathrm{mL}$ penicillin, and $100 \mu \mathrm{g} / \mathrm{mL}$ streptomycin (Solarbio, China), and filtered. Next, cells were seeded on T25 flasks containing the control medium in a humidified chamber at $5 \% \mathrm{CO}_{2}$ and $37^{\circ} \mathrm{C}$. After a 48 -hour incubation, the nonadherent cells were removed and fresh culture medium was added to the flasks. Medium was replaced every 3 days. When the cells reached $80 \%-90 \%$ confluence, adherent cells were trypsinized, harvested, and expanded. We characterized with anti-CD45 monoclonal antibody (Biolegend) as negative control and anti-CD29, CD44, and CD90 antibodies (Biolegend) as positive markers for the characterization of BM-MSCs using flow cytometry. The BM-MSCs used in the experiments were from passages 6 to 8 .

\section{BV2 microglia culture and treatment}

The BV2 murine microglial cell line was purchased from iCell Bioscience Inc., (Shanghai, China). The cells were cultured in a humidified incubator at $37^{\circ} \mathrm{C}$ with $5 \% \mathrm{CO}_{2}$ in DMEM/F12 supplemented with $10 \%$ heat-inactivated FBS, $100 \mathrm{U} / \mathrm{mL}$ penicillin, and $100 \mu \mathrm{g} / \mathrm{mL}$ streptomycin (Solarbio). BV2 cells were exposed to $10 \mu \mathrm{M}$ OxyHb (Jinpin Chemical Technology, China) to mimic SAH condition as reported previously $[18,19]$. To evaluate the effects of BMMSCs on OxyHb-stimulated microglia, we used a Costar 6-well and 24-well transwell system. BV2 cells were seeded in the bottom chamber, and BM-MSCs were seeded in the insert $(0.4 \mu \mathrm{m}$ pore size; Corning). After coculture for $24 \mathrm{~h}, \mathrm{BV} 2$ cells were isolated and processed for further evaluations.

\section{Nitric oxide (NO) release assay}

NO concentrations in culture supernatant were determined by measuring nitrite, which is a major product of NO, using a Total NO Assay Kit (Beyotime, China). Briefly, cells culture medium was collected after centrifugation at $1,000 \mathrm{rpm}$ for $10 \mathrm{~min}$. Then $50 \mu \mathrm{l}$ standard or cell culture supernatant was mixed with an equal amount of Griess reagent I and Griess reagent II and incubated at room temperature for $5 \mathrm{~min}$. The absorbance was measured at $540 \mathrm{~nm}$ on a microplate reader. Nitrite concentration was determined from a standard curve $(0-100 \mu \mathrm{mol} / \mathrm{L})$ derived from $\mathrm{NaNO}_{2}$ (Beyotime). All samples were tested in triplicate and three independent experiments were performed.

\section{$R N A$ isolation and quantification real-time polymerase chain reaction ( $q R T-P C R$ )}

After $24 \mathrm{~h}$ of treatment, the expression levels of $T N F-\alpha, I L-$ $1 \beta, I L-6, I L-10, C D 16, C D 32$, iNOS, CD80, CD86, CD206, Arg- 1 , and TGF- $\beta$ mRNA in BV2 cells were evaluated using real-time PCR. In brief, total RNA was extracted from BV2 cells using a Total mRNA Isolation Kit (TIANGEN, China) according to the manufacturer's instructions. Total RNA from each sample was reverse transcribed using PrimeScript ${ }^{\mathrm{TM}} \mathrm{RT}$ reagent Kit with gDNA Eraser (TAKARA). qRT-PCR was conducted using an Analytikjena qPOWER real-time PCR system (JENA, German) with QuantiNova SYBR Green PCR Kit (QIAGEN, German) according to the manufacturer's instructions. The reactions were performed in a total volume of $20 \mu \mathrm{l}$ containing $0.4 \mathrm{mM}$ of each primer (Table 1). Glyceraldehyde-3-phosphate dehydrogenase $(G A P D H)$ was employed as the endogenous control. Triplicate measurements were performed for all reactions. The results were analyzed using the comparative critical threshold $(\triangle \Delta \mathrm{CT})$ methods in the qPCR software program.

\section{Western blotting analysis}

The total proteins were extracted using RIPA lysis buffer (Beyotime) with a protease inhibitor cocktail (Beyotime). Cell lysates were then centrifuged at $12,000 \mathrm{~g}\left(4^{\circ} \mathrm{C}\right)$ for $15 \mathrm{~min}$ and the supernatant was collected to determine protein concentration using a bicinchoninic acid assay kit (Beyotime). Forty micrograms of protein extract from each sample was separated by sodium dodecyl sulfate-polyacrylamide gel electrophoresis and then transferred onto a nitrocellulose membrane $(0.45 \mu \mathrm{m}$; Millipore) using a wet transfer system. The membranes were blocked using 5\% nonfat dry milk in Tris-buffered saline and Tween 20 and then incubated with primary antibodies directed against CD16 (ABclonal; 1:1,000), CD86 (ABclonal; 1:1,000), CD206 (ABclonal; 1:1,000), Arg-1 (Cell Signal Technology; 
Table 1. List of Primers Used in Quantification Real-Time Polymerase Chain Reaction Studies

\begin{tabular}{lll}
\hline Gene name & \multicolumn{1}{c}{ Forward sequence } & \multicolumn{1}{c}{ Reverse sequence } \\
\hline$G A P D H$ & GGACCTCATGGCCTACATGG & TAGGGCCTCTCTTGCTCAGT \\
$T N F-\alpha$ & ATGTCTCAGCCTCTTCTCATTC & GCTTGTCACTCGATTTTTGAGA \\
$I L-1 \beta$ & CAACTGTTCCTGAACTCAACTG & GAAGGAAAAGAAGGTGCTCATG \\
$I L-6$ & TACCACTTCACAAGTCGGAGGC & CTGCAAGTGCATCATCGTTGTTC \\
$C D-10$ & CGGGAAGACAATAACTGCACCC & CGGTTAGCAGTATGTTGTCCAGC \\
$C D 32$ & TATGGCACCTTAGCGTGATG & CGACCCTGTAGATCTGGGAG \\
$i N O S$ & AATCCTGCCGTTCCTACTGATC & GTGTCACCGTGTCTTCCTTGAG \\
$C D 86$ & GCTTGTCTCTGGGTCCTCTG & CTCACTGGGACAGCACAGAA \\
$C D 206$ & CCTCAAGTTTCCATGTCCAAGGC & GAGGAGAGTTGTAACGGCAAGG \\
$A r g-1$ & TCTCCACGGAAACAGCATCT & CTTACGGAAGCACCCATGAT \\
$T G F-\beta$ & TCAGCTATTGGACGCGAGGCA & TCCGGGTTGCAAGTTGCCGT \\
& TTAGGCCAAGGTGCTTGCTGCC & TACCATGGCCCTGAGGAGGTTC \\
\hline
\end{tabular}

1:1,000), and GAPDH (Proteintech; $1: 4,000)$ at $4^{\circ} \mathrm{C}$ overnight. The secondary antibodies were applied for $1 \mathrm{~h}$ at room temperature. The immunoblots were visualized using enhanced chemiluminescence (ECL; Fdbio Science). Digital images were sequentially analyzed by ImageJ to obtain the optical densities (OD) of signals, which were semiquantified and expressed as a ratio of the OD of the tested proteins to the OD of the control, GAPDH.

\section{Immunofluorescence staining}

BV2 cells were seeded in the bottom chamber of 24-well transwell system with glass coverslips, cocultured with BMMSCs in the presence or absence of OxyHb $(10 \mu \mathrm{M})$ for $24 \mathrm{~h}$. Then the cells were washed using PBS and fixed using $4 \%$ paraformaldehyde at room temperature. Following three 5-min washes in PBS, the cells were blocked with 5\% goat serum in PBS for 30 min before incubation with primary antibodies against Iba1 (Abcam; 1:200) and CD16/32 (BD Pharmingen; 1:200) or CD206 (Abcam; 1:200) at $4^{\circ} \mathrm{C}$ overnight. The following day, cells were incubated with the secondary antibodies Alexa Fluor 594 goat anti-rabbit IgG and Alexa Fluor 488 goat anti-rat IgG or Alexa Fluor 488 goat anti-mouse IgG (Proteintech; 1:200) for $1 \mathrm{~h}$ at room temperature in dark. After three washes again, the coverslips were counterstained by 4,6-diamidino-2-phenylindole (DAPI; Solarbio) for $15 \mathrm{~min}$ at room temperature. Images were obtained with Olympus FV1000 confocal microscope (Olympus, Tokyo, Japan) under the same exposure time.

\section{RNA-seq library construction and RNA-seq}

Total RNA was extracted from each group of BV2 cells (control, OxyHb, OxyHb with BM-MSCs coculture) using TRIzol (Life Technologies) according to the manufacturer's instructions. Five hundred nanograms of total RNAs was used as startup input for each sequencing library construction (ABclonal). All purified libraries were sequenced on Illumina HiSeq $\mathrm{X}$ Ten (Illumina, San Diego, CA) to acquire $150 \mathrm{bp}$ paired-end sequence reads, at Novogene Corporation (Nanjing, China). Three replicates of BV2 cells for each group were sequenced.

\section{Sequencing data analysis}

RNA-seq data analysis procedures include raw read processing, clean read mapping and filtering, and gene expression level normalization. For raw read processing, library adaptors were removed from raw reads first, using trim_galore tool (0.4.4_dev). The last five bases and reads of mean quality value across each base position $<20$ were trimmed until matching the quality threshold by trim_galore tool. Then clean paired reads were aligned to mouse genome (GRCm38; Ensembel) using HISAT2 with default parameters. The unmapped reads were filtered out by samtools (v1.7).

Gene ontology (GO) and biological pathways and functions were determined using the web-based bioinformatics software Ingenuity Pathway Analysis (IPA; Ingenuity Systems). A fold change of \pm 2 in expression levels and $P<0.05$ were used as a cutoff to generate data sets of significantly differential expressed genes. To further analyze the biological impact of the differentially expressed genes (DEGs), we created proteinprotein interaction networks using the STRING database.

\section{Statistical analysis}

The statistical analysis was performed using Graph Pad Prism (Graph Pad Software, San Diego, CA). Each value is expressed as the mean and standard deviation (mean $\pm \mathrm{SD}$ ). One-way analysis of variance was used followed by multiple comparisons between groups using Tukey's honest significant difference (HSD) post hoc test and Holm-Bonferroni correction. $P<0.05$ was considered statistically significant.

\section{Results}

\section{Identification and labeling of BM-MSCs}

BM-MSCs were obtained from the bone marrow of C57BL/6J mice as mentioned in the materials. Passage 4 BM-MSCs adhered to the bottom of the culture dishes and grew with a fibroblast-like morphology (Fig. 1A). Flow cytometry was used to characterize the BM-MSCs by detecting surface antigen expression. BM-MSCs were positive for CD29 (96.7\%), CD90 (91.1\%) and CD44 (71.0\%), but were negative for CD45 (3.59\%) (Fig. 1B).

\section{BM-MSCs coculture ameliorated OxyHb-induced microglial activation}

An in vitro model of SAH was established by exposing BV2 cells to $\mathrm{OxyHb}$ at a concentration of $10 \mu \mathrm{M}$. Morphological changes and enhanced NO production are the hallmarks of 
A

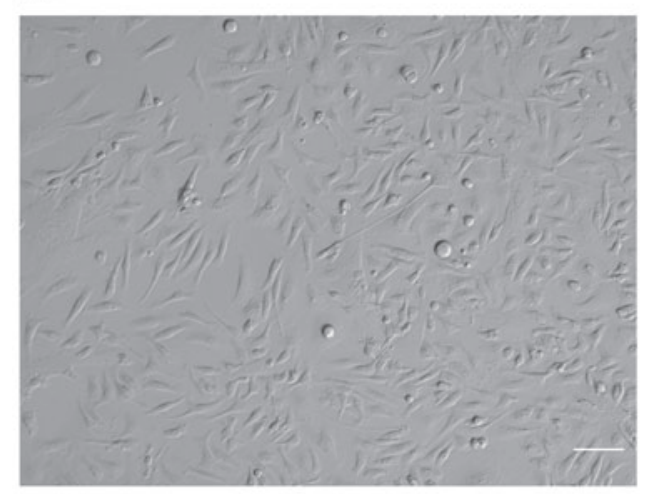

B
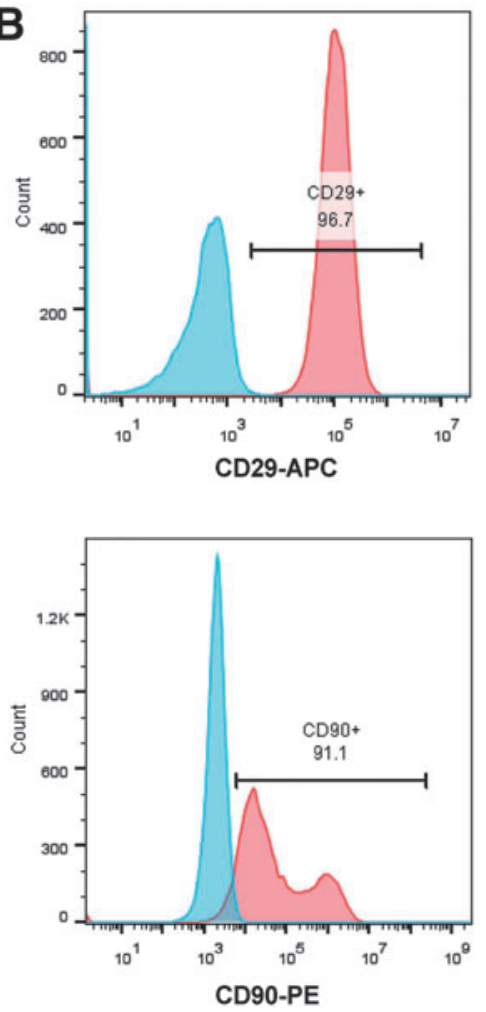
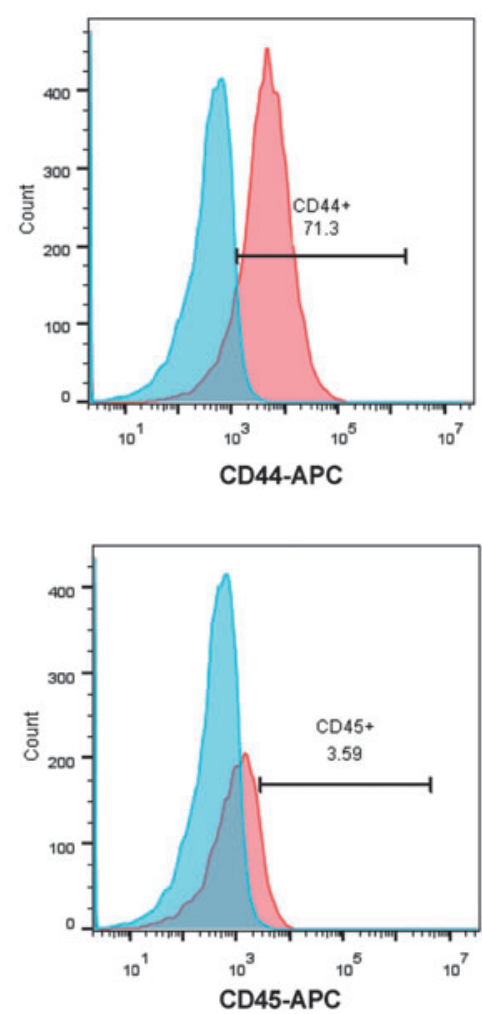

FIG. 1. Isolation, characterization, and labeling of BM-MSCs. (A) The observation of the passage 4 BM-MSCs by a light microscope. Scale bar $=50 \mu \mathrm{m}$. (B) Flow cytometric analysis showing the expression levels of CD29, CD44, CD90, and CD45 in the passage 4 of BM-MSCs. BM-MSCs, bone marrow-derived mesenchymal stem cells.

microglial activation [20-22]. We found that OxyHb stimulation induced $\mathrm{NO}$ release in a time-dependent manner and peaked at $24 \mathrm{~h}$ (Fig. 2A). Importantly, BM-MSC coculture suppressed the production of NO by $\sim 30 \%$ (Fig. 2B). We also observed the morphological changes occurring in microglial activation following treatment with $\mathrm{OxyHb}$. As shown in Fig. 2C, in the control group, microglia exhibited a resting type manifested by small cell bodies and elongated processes. Upon OxyHb stimuli, the processes of microglia retracted and thickened, which indicated the microglial activation. Consistent with the effect on NO production, BM-MSCs coculture significantly attenuated the activated morphology of microglia (Fig. 2C).

\section{BM-MSCs coculture modulated OxyHb-induced inflammatory gene expression and microglial M1 polarization}

Next, we investigated the effect of BM-MSCs on the expression of inflammatory factors in OxyHb-activated microglia. To this end, BV2 microglial cells were treated with $\mathrm{OxyHb}$ for $24 \mathrm{~h}$ in the presence or absence of BMMSCs. As shown in Fig. 3A, OxyHb stimulation increased the expression level of proinflammatory genes, including $T N F-\alpha, I L-6$, and $I L-1 \beta$, indicating the presence of a strong inflammation response. Coculture of BM-MSCs reduced the expression level of $T N F-\alpha$ and $I L-1 \beta$, but not $I L-6$, suggesting that the effect of BM-MSCs on the expression of inflammatory genes in BV2 cells is highly selective. In contrast, BM-MSCs coculture upregulated the expression of anti-inflammatory gene $I L-10$.
Following SAH, activated microglia become polarized into two distinct phenotypes, broadly known as proinflammatory M1 and anti-inflammatory M2 phenotypes [23-28]. To further detect whether BM-MSCs regulated microglial polarization directly, we explored the phenotype of microglia by measuring the expression of various M1/M2 markers under different treatment. qRT-PCR analysis revealed that the expression level of M1 markers (CD16, CD32, iNOS, CD80, and CD86) was strongly upregulated by OxyHb stimulation, which was significantly attenuated by BM-MSCs coculture (Fig. 3B). Although M2 markers (CD206, Arg-1, and TGF- $\beta$ ) were greatly suppressed by $\mathrm{OxyHb}$ treatment, BM-MSCs did not alter the gene expression levels of the M2 markers. Consistently, western blot results showed similar alterations at protein level that treatment of BM-MSCs reduced the enhanced M1 marker (CD16 and CD86) signal, while the signal of M2 markers (CD206 and Arg-1) was unaffected by BMMSC coculture (Fig. 3C). The results were further confirmed by immunofluorescent staining with Iba-1 and M1 marker CD16/32 or M2 marker CD206 (Fig. 3D). These results suggested that BM-MSCs mainly modulated microglial M1 polarization rather than M2 polarization.

\section{Inflammation-related transcriptomic changes in OxyHb-stimulated BV2 cells cocultured with BM-MSCs}

To gain a comprehensive understanding of the mechanisms involved in the mediation of OxyHb-induced microglial inflammation by BM-MSCs, RNA-seq was utilized. The subsequent functional analysis was performed to identify 

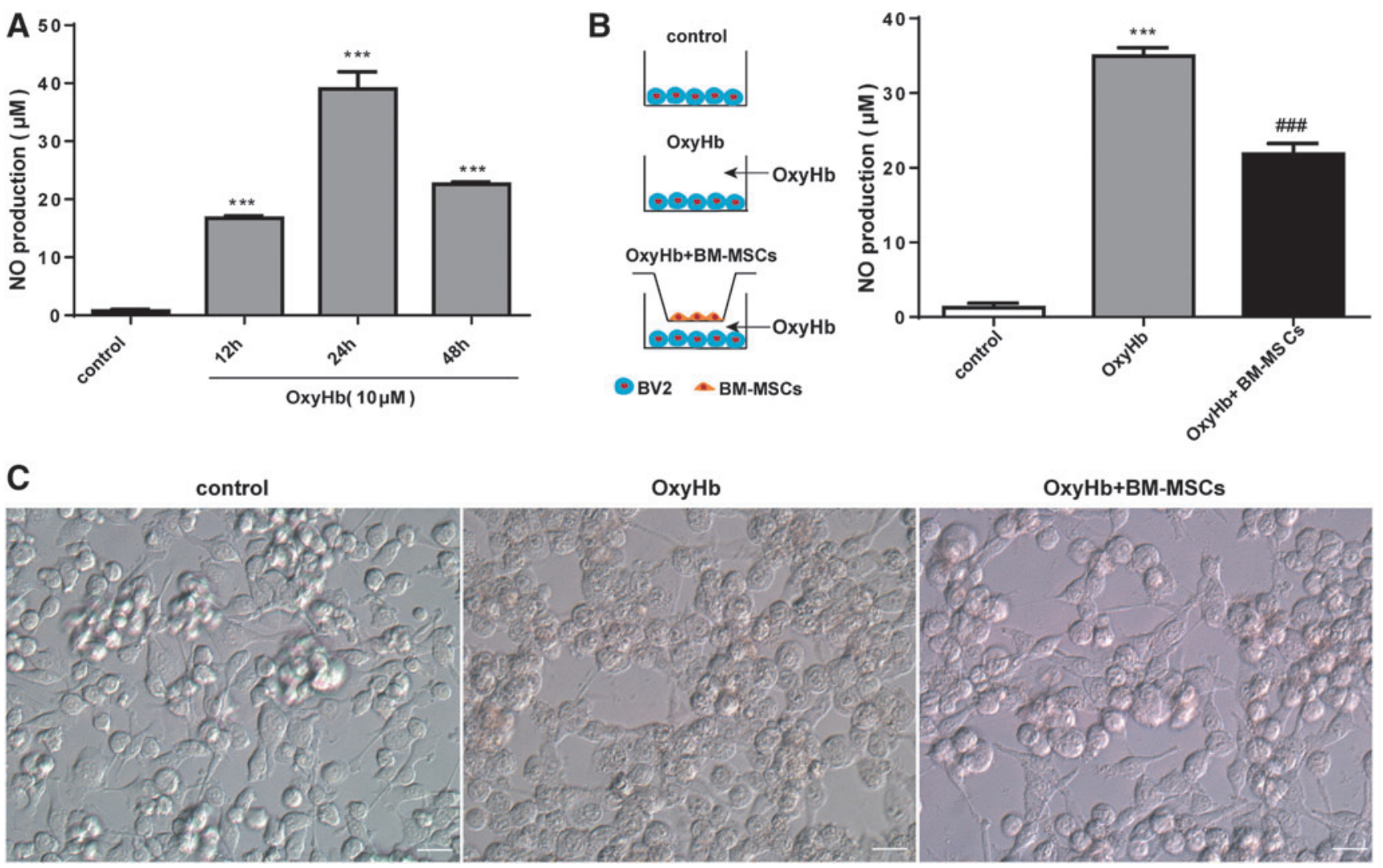

FIG. 2. Effects of BM-MSCs coculture on OxyHb-induced NO production of BV2 cells. (A) Time course of OxyHbinduced NO production of BV2 cells; (B) in vitro coculture experimental design and the inhibitory effect of BM-MSCs coculture on OxyHb-induced NO production of BV2 cells; (C) cellular morphology of BV2 cells under different treatment. Scale bar $=100 \mu \mathrm{m}$. Values are mean \pm SD. $n=3$; *** $P<0.001$ versus control, \#\#\# $P<0.001$ OxyHb+BM-MSCs versus $\mathrm{OxyHb}$. NO, nitric oxide; OxyHb, oxyhemoglobin.

differentially expressed genes DEGs and regulatory pathways between OxyHb-stimulated BV2 cells cocultured with and without BM-MSCs. GO analysis revealed that most of the DEGs were enriched in intracellular membrane-bound organelle, intracellular part and cellular process (Fig. 4A), suggesting that BM-MSCs coculture induced an overall change of cellular component and signal transduction in BV2 cells. Next, we compared the DEGs with the Kyoto encyclopedia of genes and genomes (KEGG) pathway database to predict which biological pathways the identified genes participate in. The result showed that the DEGs were highly clustered in several signaling pathways, especially FoxO signaling pathway, TNF signaling pathway, and PI3K-Akt signaling pathway (Fig. 4B), which were related to the regulation of inflammation response [29-32].

For detailed transcriptomic analysis of the inflammatory response, we selected genes related to inflammatory response with altered expression. Heat map analysis showed remarkable changes in 91 genes between the presence and absence of BM-MSCs in OxyHb-treated BV2 cells (Fig. 5A). To further investigate the interactions among the differentially expressed inflammatory response-related genes, we investigated connectivity among the identified genes by assessing published physical and genetic interactions using the online bioinformatics tool, String (version 11.0). The networks showed that the molecules Akt1 and $I L 1 \beta$ were predicted as the important hub nodes (Fig. 5B).

\section{Identification of novel epigenetic regulators in OxyHb-induced BV2 cells cocultured with BM-MSCs}

Epigenetic regulation, which involves DNA methylation and histone modifications, is emerging as one of the major mechanisms regulating gene expression in response to environmental stimuli [33,34]. The annotation of the RNA-seq data revealed family-wide DEGs implicated in epigenetic regulation. Herein, we provide the first evidence that among multiple families of epigenetic regulators, only histone demethylase-related genes $K d m 6 b$ and $K d m 7 a$ were significantly differentially expressed after $\mathrm{OxyHb}$ stimulation in BV2 cells (Fig. 6). It should be noted that BM-MSC coculture significantly increased the expression of Kdm6b, which was decreased in OxyHb-stimulated BV2cells, suggesting that $K d m 6 b$ might play a critical role in the regulation of OxyHb-induced microglial inflammation. Besides that, BM-MSCs coculture also significantly upregulated the expression of DNA methyltransferase gene Dnmt3l-ps1, histone deacetylase Hdac5, and histone methyltransferase gene Setd7.

\section{Discussion}

SAH represents a life-threatening cerebrovascular disease with high mortality and morbidity rates. Recent studies have 

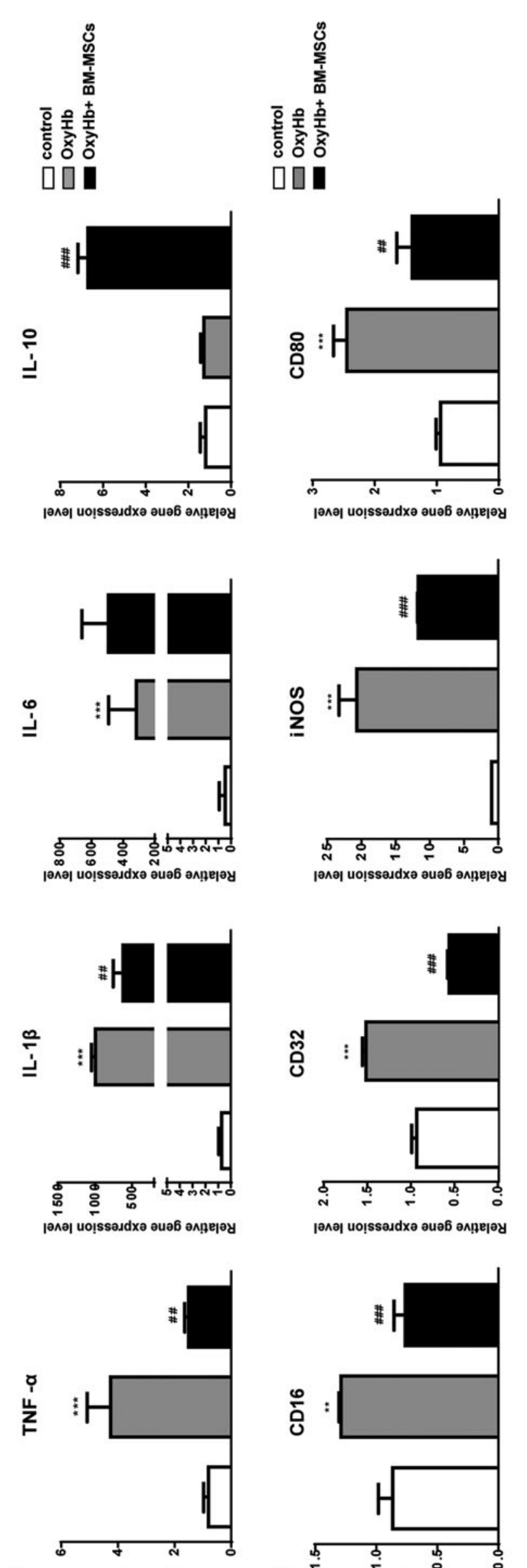

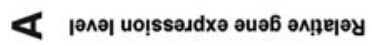

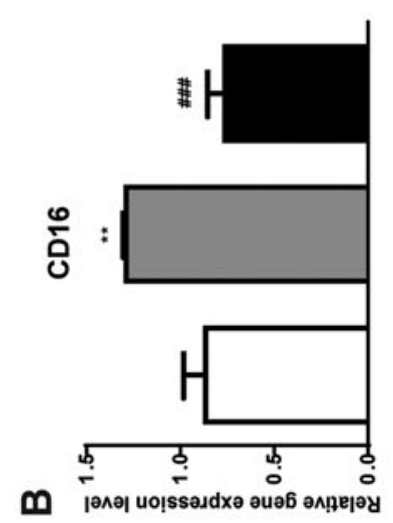

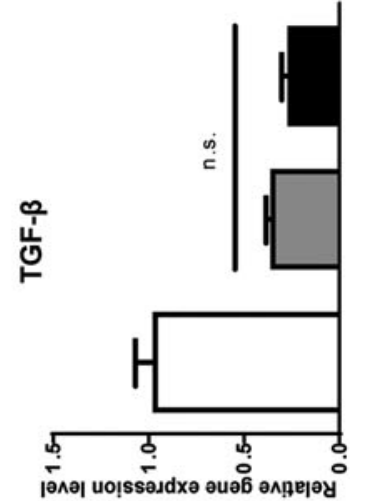
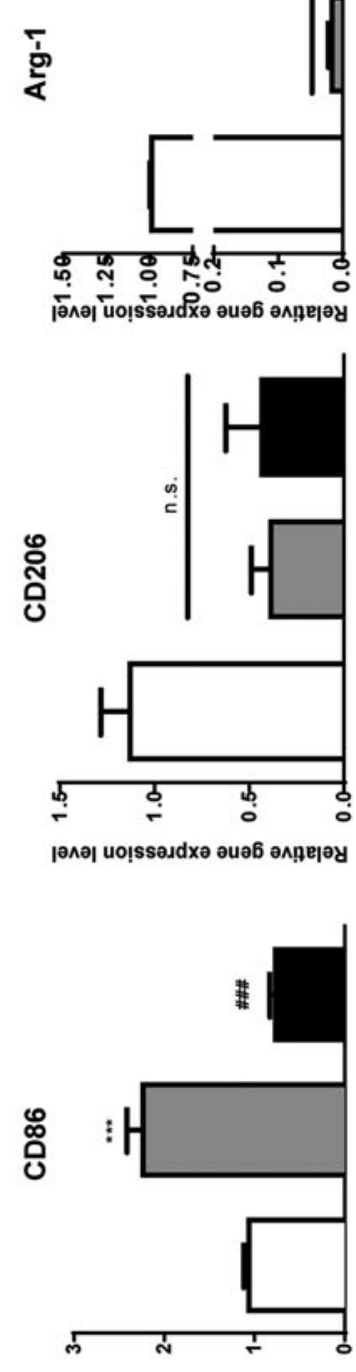

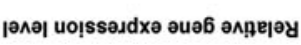

Q

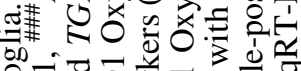

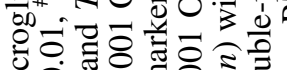

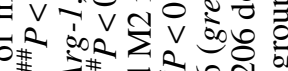

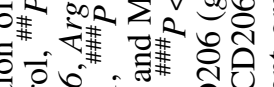

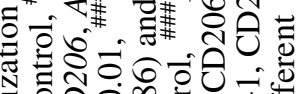

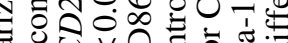

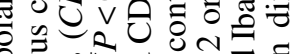

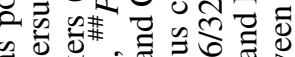
\%

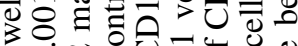

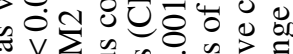

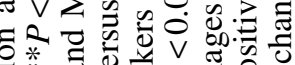

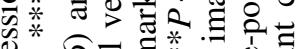
mis ख $\|$ : $0 . \Sigma^{*}$ m i v $v$ प II एक जै ₹ $\approx 0$ के $+10 \%$ : 0130

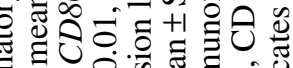
घ0 0 . छ

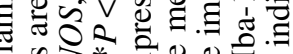

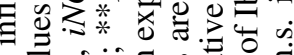

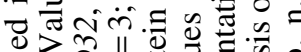

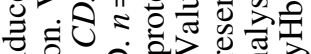

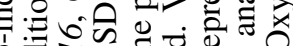

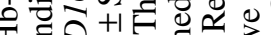

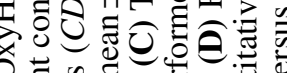
o $n$ 四

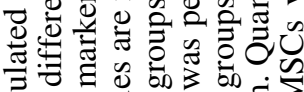

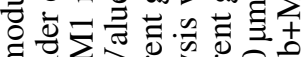
छ $\sum_{4}$

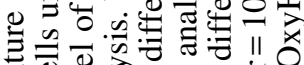
릉 $\overline{0} \approx 0$

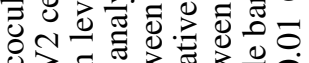
伩零

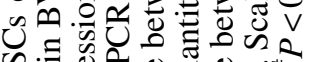
2.

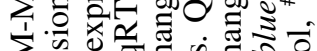

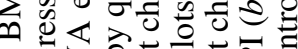

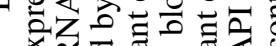

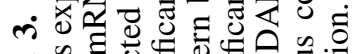

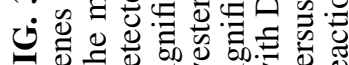




\section{总

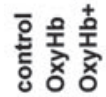 \\ 미}

号
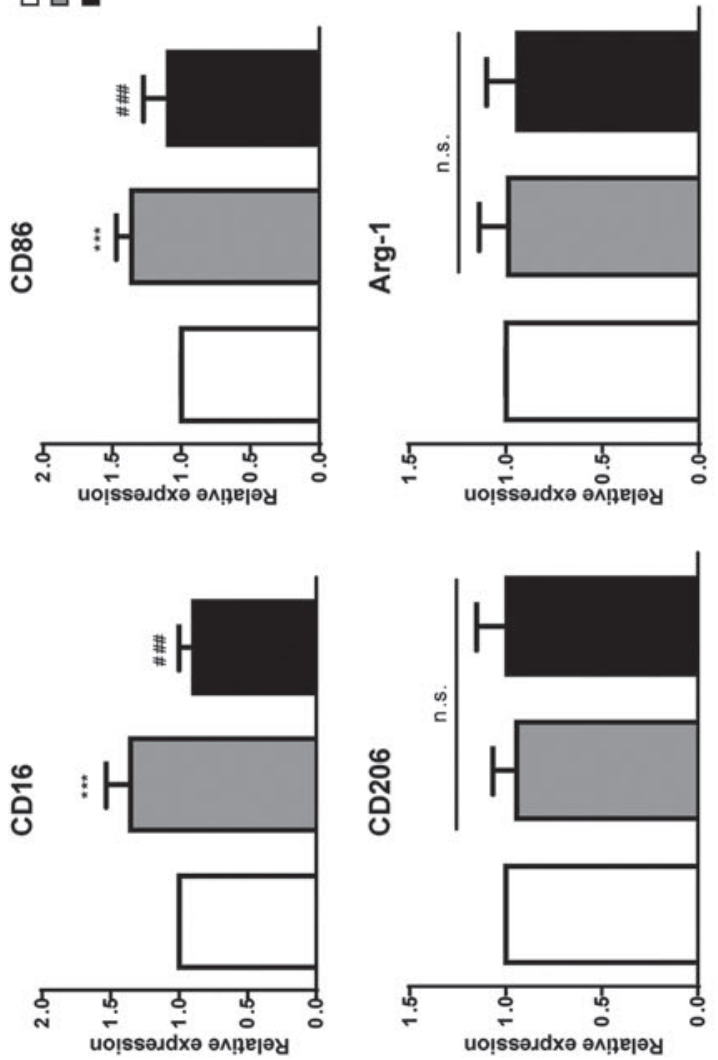
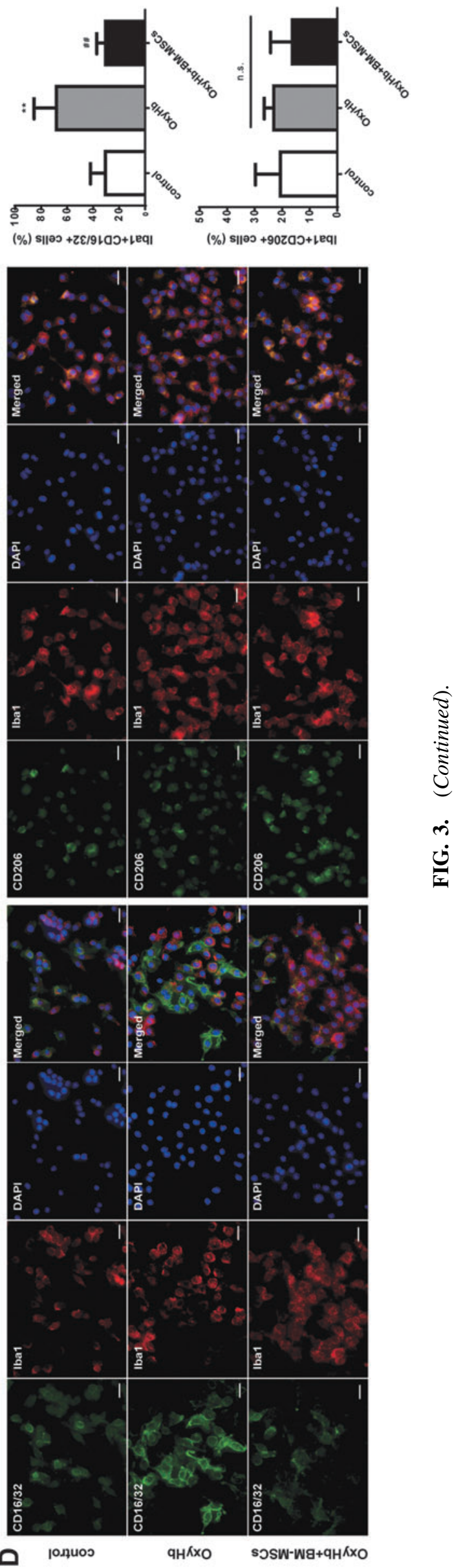

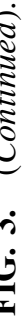

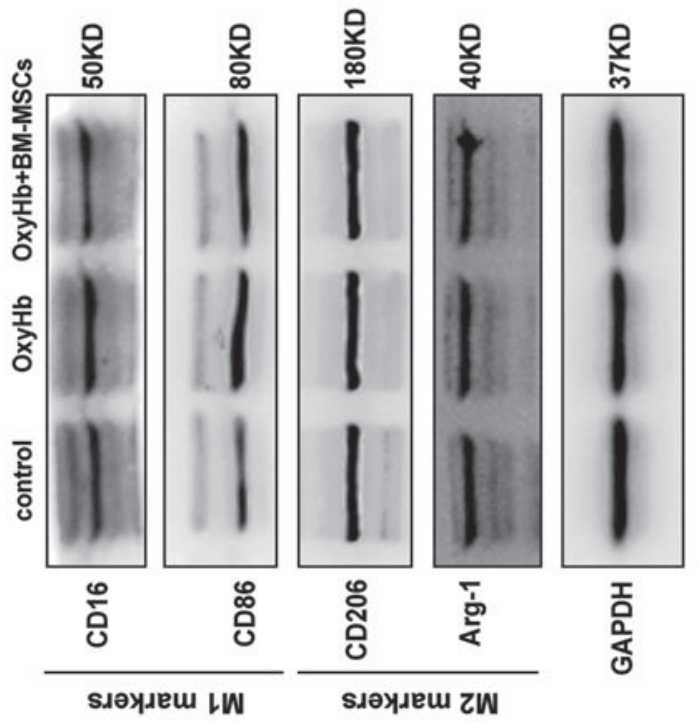



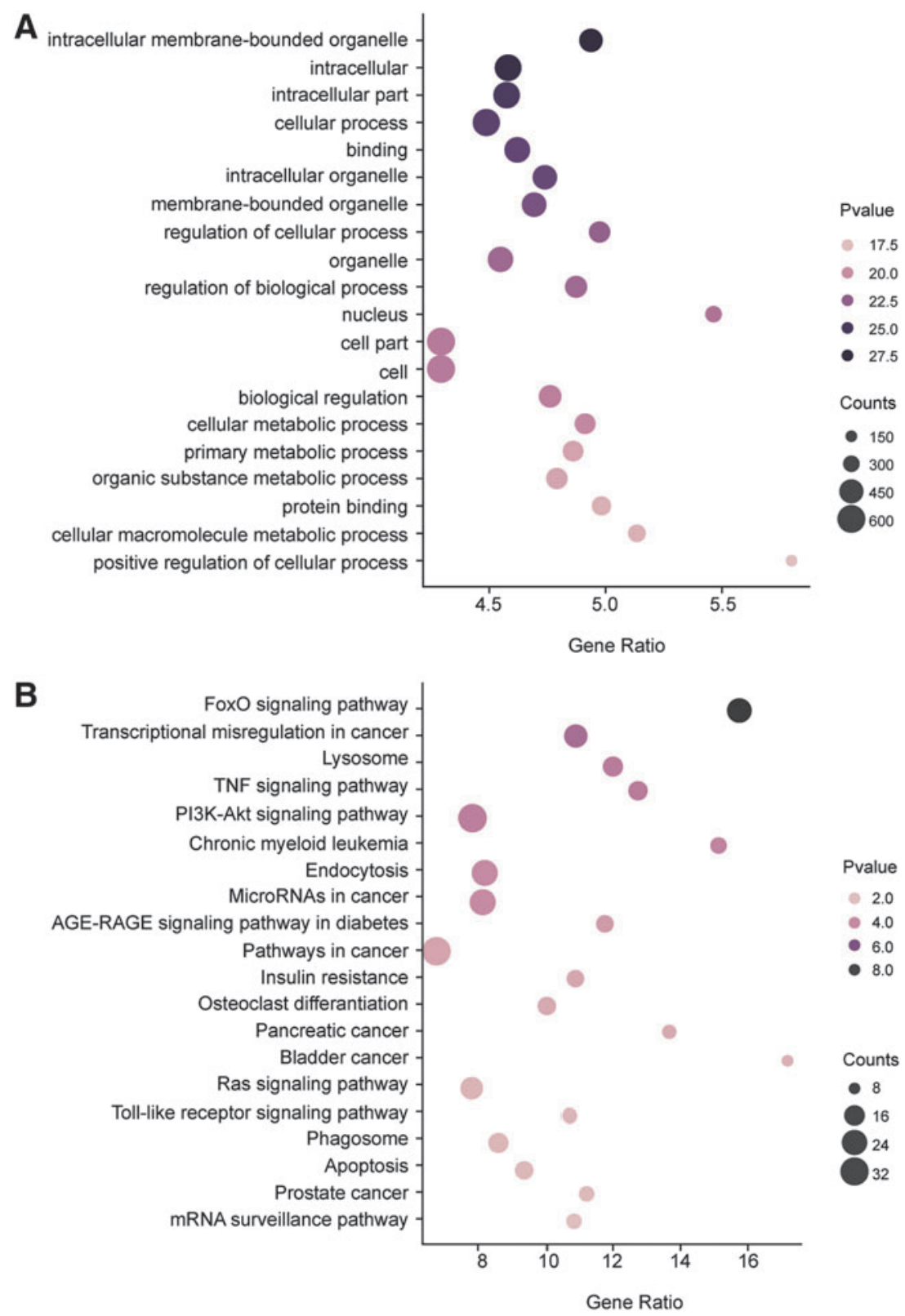

FIG. 4. GO analysis and KEGG assay for differentially expressed genes in OxyHb-stimulated BV2 cells cocultured with BM-MSCs compared to OxyHbstimulated BV2 cells. (A) Representation of the results obtained after a standard functional enrichment analysis of differentially expressed genes using GO terms. The size of the circles is proportional to the number of genes in that category, reflecting differential expression, while color indicates $P$-value. (B) Scatter plot of top 20 pathways that are associated with the differentially expressed coding genes. Vertical coordinate represents pathway name, and horizontal coordinate represents rich factor. The size and color of points represent the number of differentially expressed genes in the pathway and the range of different corrected $P$-value, respectively. GO, gene ontology; KEGG, Kyoto encyclopedia of genes and genomes. shown that EBI after SAH seems to contribute to the poor outcome of SAH patients [3,4]. It is indicated that microgliamediated neuroinflammation is an underlying key player in the pathogenesis of SAH-induced EBI $[5,26]$. Upon sensing a disturbance in homeostasis after $\mathrm{SAH}$, microglia are activated and polarized to M1 microglia, resulting in the release of proinflammatory mediators. Therefore, modulating microglial activation and polarization appears to be an attractive approach to alleviate inflammation-induced EBI after SAH $[9,23]$. One such experimental method beginning to emerge is the use of stem cell therapy. Exogenously transplanted BMMSCs migrated to damaged tissue sites, where they differentiated into several different lost or injured cell types and modulate immune responses $[35,36]$. Recently, it was reported that BM-MSCs transplantation ameliorated brain edema, neurobehavioral deficits, and neuronal injury due to the inhibition of microglia activation and decreased expression of inflammatory cytokines post-SAH $[16,17]$. However, detailed transcriptomic mechanisms of how BM-MSCs modulate microglia-mediated neuroinflammation post-SAH are not well understood.

SAH is characterized as extravasation of blood into the subarachnoid space. Hemoglobin, which leaks from damaged erythrocytes, is a key factor to the neuroinflammation after SAH $[37,38]$. Therefore, we applied $\mathrm{OxyHb}$ stimulation to mimic the SAH conditions as previously reported $[18,19]$. To evaluate the effects of BM-MSCs on OxyHb-stimulated microglia, BM-MSCs and BV2 were cocultured through a Costar transwell system. This study showed that OxyHb treatment induced the production of NO, a hallmark of microglial activation. Consistently, the expression of proinflammatory factors was also enhanced by $\mathrm{OxyHb}$ treatment. Importantly, BM-MSCs coculture attenuated the OxyHb-mediated microglial activation as demonstrated by the suppression of NO release as well as the expression of TNF- $\alpha$ and IL- $1 \beta$. On the other hand, 
A

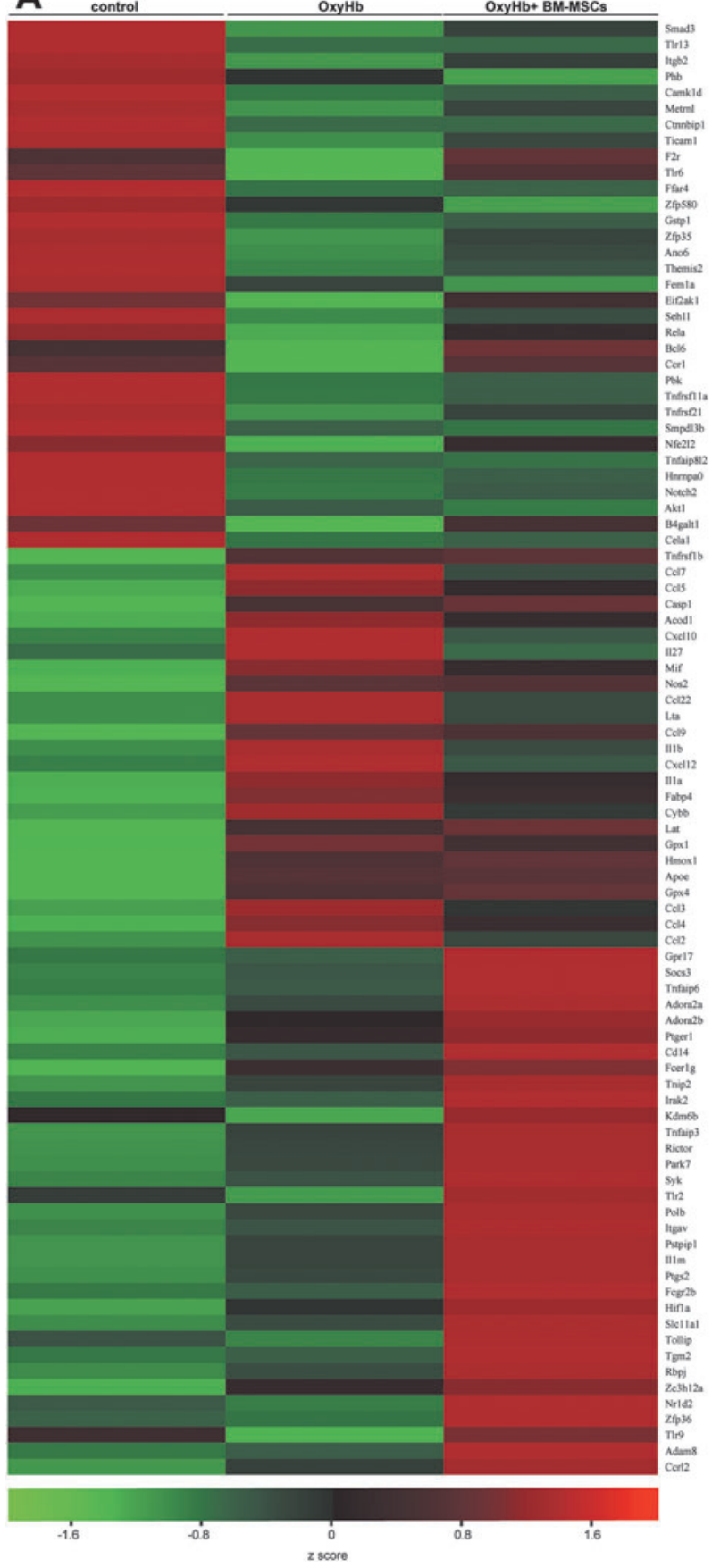

B

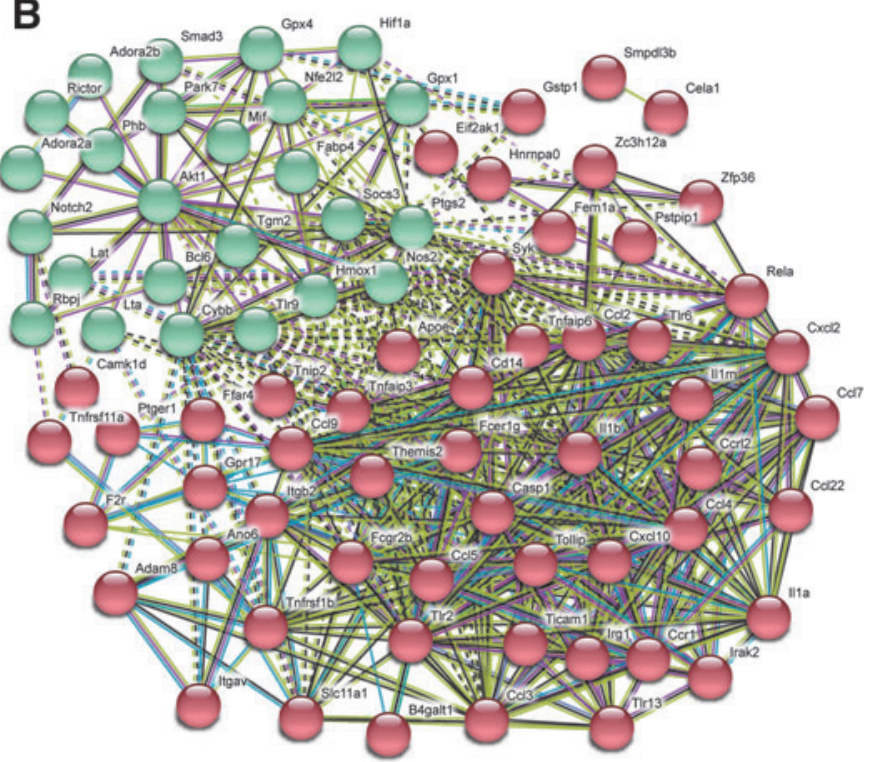

FIG. 5. Inflammation-related gene expression variation in OxyHb-stimulated BV2 cells cocultured with BM-MSCs. (A) Heat map of genes related to inflammation with altered expression levels in BV2 cells stimulated with OxyHb in the presence or absence of BM-MSCs coculture. Gene expression values are colored from green (downregulated) to red (upregulated). (B) Gene network related to inflammatory response was constructed. Network is clustered to a specified number of clusters.

coculture of BM-MSCs enhanced the expression of antiinflammatory gene IL-10. Further study demonstrated that BM-MSCs regulate OxyHb-induced microglial M1 polarization rather than $\mathrm{M} 2$ polarization, because coculture of BM-MSCs weakened the characteristics of M1 polarization without any influence on the M2 markers.

It should be noted that although BM-MSCs treatment reduced OxyHb-meditated upregulation of microglial proinflammatory factors TNF- $\alpha$ and IL- $1 \beta$, the expression of IL-6 remains unchanged upon BM-MSCs coculture. In fact, it has been recognized that IL-6 is a pleiotropic cytokine with proinflammatory and anti-inflammatory properties. In classic signaling, IL-6 stimulates target cells through a membrane-bound IL-6 receptor, which upon ligand binding associates with the membrane glycoprotein gp130 [18]. However, only few cells express membranebound IL-6 receptor. Cells that express only gp130 respond to a complex of IL- 6 bound to a naturally occurring soluble 

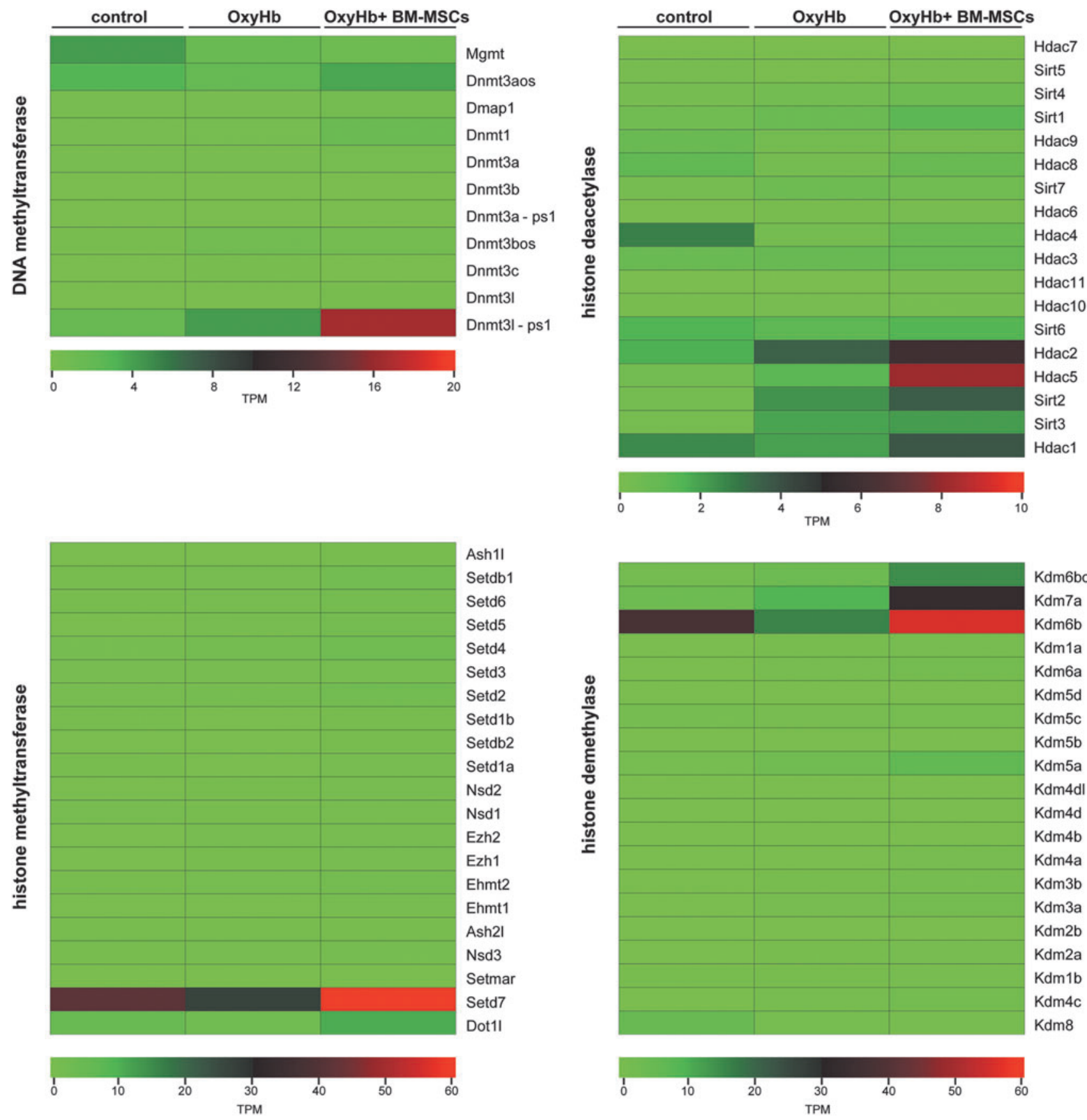

FIG. 6. Epigenetic mechanisms for the regulation of OxyHb-induced microglial inflammation by BM-MSCs. The heat maps display family-wide collections of genes encoding DNA/histone methyltransferases, histone deacetylases, and histone demethylases.

form of IL-6 receptor, which is termed trans-signaling. It turns out that regenerative or anti-inflammatory activities of IL-6 are mediated by classic signaling, whereas proinflammatory responses of IL-6 are rather mediated by transsignaling. Importantly, it has been reported that BV2 cells expressed both gp130 and IL-6R on the cell surface [39], indicating that IL-6 has both proinflammatory and antiinflammatory effects in BV2 cells. This may explain why $\mathrm{BM}-\mathrm{MSCs}$ have little effect on the OxyHb-induced expression of IL-6 in BV2 cells.
To elucidate the detailed mechanism of how BM-MSCs downregulated OxyHb-induced microglial activation, we implemented RNA-seq approaches to characterize global transcriptional responses in OxyHb-infected BV2 cells cocultured with BM-MSCs. A total of 772 DEGs were identified and GO analysis revealed that most of DEGs were categorized into "biological process," suggesting that BMMSCs coculture induced an overall change of signal transduction in OxyHb-stimulated BV2 cells. KEGG mapping identified several pathways that were highly enriched, 
especially FoxO signaling pathway, TNF signaling pathway, and PI3K-Akt signaling pathway. As predicted, we further found that the expression of inflammatory response-related genes was strongly altered by BM-MSCs coculture. These phenomena may occur as a result of secreted molecules from BM-MSCs; further studies are needed to identify the secreted molecules, which suppress the inflammatory response of OxyHb-stimulated microglia.

In addition to the inflammation signaling pathway, annotation of DEGs in our RNA-seq data revealed differential expression of genes involved in epigenetic regulation, which is defined as nongenetic alterations critical to biological adaptability to environmental stimuli. Herein, we provide the first evidence that among multiple families of epigenetic regulators, only histone demethylase-related genes $K d m 6 b$ and $K d m 7 a$ were significantly differentially expressed after $\mathrm{OxyHb}$ stimulation in BV2 cells. It was worth noting that the downregulated expression of $K d m 6 b$ caused by $\mathrm{OxyHb}$ stimulation was recovered by BM-MSCs coculture, suggesting the critical role of $k d m b b$ in the regulation of $\mathrm{OxyHb}$-induced BV2 microglial inflammation.

$\mathrm{Kdm} 6 \mathrm{~b}$ is an inducible histone demethylase, which potentiates gene expression by demethylating repressive $\mathrm{H} 3 \mathrm{~K} 27 \mathrm{me} 3$ epigenetic marks in promoters and gene bodies. Recent studies on the molecular biology of Kdm6b have revealed that the expression of Kdm6b can be induced by many inflammatory mediators as well as stress inducers, e.g., metabolic, hypoxic, and oncogenic stress. Kdm6b can enhance both proinflammatory and anti-inflammatory responses by targeting distinct transcription factors in a context-dependent manner in gene promoters [40,41]. Researchers demonstrated that the LPS treatment of bone marrow-derived macrophages triggered a clear recruitment of $\mathrm{Kdm} 6 \mathrm{~b}$ to the transcription start sites of thousands of activated genes, and the deletion of Kdm6b impaired the transcription of numerous inflammatory genes [42], while in Parkinson's disease, suppression of Kdm6b augments the M1 proinflammatory response by inhibiting M2 microglia polarization and leading to neuronal cell death [43]. According to our result, we hypothesized that in BV2 cells, endogenous Kdm6b may mainly involve in the enhancement of the anti-inflammatory responses; as a result, BM-MSCs-induced expression of Kdm6b alleviates the inflammation in OxyHbstimulated BV2 cells. Further study is needed to identify downstream effectors of Kdm6b and to identify which genes are essential for mediating Kdm6b responses.

In conclusion, this study demonstrated that BM-MSCs coculture reduced microglial activation as well as inflammatory response in OxyHb-stimulated BV2 cells. The RNA-seq elucidated the transcriptomic changes of OxyHb-stimulated BV2 cells cocultured with BM-MSCs, containing highly enriched DEGs involved in inflammatory response. We further found that the histone demethylase Kdm6b may play a critical role in the regulation of $\mathrm{OxyHb}$-induced inflammation by BM-MSCs. However, further studies have to be conducted to confirm these effects in vivo using animal model of SAH. Our analysis gives novel insights to uncover the molecular mechanism of BMMSCs treatment for SAH and may offer a new therapeutic strategy using stem cell therapy for SAH.

\section{Author Disclosure Statement}

No competing financial interests exist.

\section{Funding Information}

This work was supported by the National Natural Science Foundation of China (81771278, 81801176, and 81971132), Sichuan Science and Technology Program (2018RZ0090, 19MZGC0003, and 19CXTD0008), Luzhou Science and Technology Program (2016LZXNYD-Z02, 2018-JYJ-42), and $\mathrm{PhD}$ Research Startup Foundation of The Affiliated Hospital of Southwest Medical University.

\section{References}

1. Macdonald RL and TA Schweizer. (2017). Spontaneous subarachnoid haemorrhage. Lancet 389:655-666.

2. Allen NJ and C Eroglu. (2017). Cell biology of astrocytesynapse interactions. Neuron 96:697-708.

3. Fujii M, J Yan, WB Rolland, Y Soejima, B Caner and JH Zhang. (2013). Early brain injury, an evolving frontier in subarachnoid hemorrhage research. Transl Stroke Res 4: 432-446.

4. Cahill J, JW Calvert and JH Zhang. (2006). Mechanisms of early brain injury after subarachnoid hemorrhage. J Cereb Blood Flow Metab 26:1341-1353.

5. De Oliveira Manoel AL and RL Macdonald. (2018). Neuroinflammation as a target for intervention in subarachnoid hemorrhage. Front Neurol 9:292.

6. Adriano A, BA Barres and ML Bennett. (2013). Microglia: scapegoat, saboteur, or something else? Science 339:156-161.

7. Aloisi F. (2001). Immune function of microglia. Glia 36: $165-179$.

8. Serge R. (2009). Regulation of innate immune responses in the brain. Nat Rev Immunol 9:429.

9. Lan X, X Han, Q Li, QWYang and J Wang. (2017). Modulators of microglial activation and polarization after intracerebral haemorrhage. Nat Rev Neurol 13:420.

10. Kroner A, A Greenhalgh, J Zarruk, R Passosdossantos, M Gaestel and S David. (2015). TNF and increased intracellular iron alter macrophage polarization to a detrimental M1 phenotype in the injured spinal cord. Neuron 86:1317.

11. Miron VE, B Amanda, Z Jing-Wei, TJ Yuen, JM Ruckh, JL Shadrach, VW Peter, AJ Wagers, W Anna and RJM Franklin. (2013). M2 microglia and macrophages drive oligodendrocyte differentiation during CNS remyelination. Nat Neurosci 16:1211-1218.

12. Parr AM, CH Tator and A Keating. (2007). Bone marrowderived mesenchymal stromal cells for the repair of central nervous system injury. Bone Marrow Transplant 40:609-619.

13. Uccelli A, L Moretta and V Pistoia. (2008). Mesenchymal stem cells in health and disease. Nat Rev Immunol 8:726736.

14. Napoli E and CV Borlongan. (2016). Recent advances in stem cell-based therapeutics for stroke. Transl Stroke Res 7:452-457.

15. Rodríguez-Frutos B, L Otero-Ortega, M GutiérrezFernández, B Fuentes, J Ramos-Cejudo and E Díez-Tejedor. (2016). Stem cell therapy and administration routes after stroke. Transl Stroke Res 7:378-387.

16. Nijboer CH, E Kooijman, CT Van Velthoven, E Van Tilborg, IA Tiebosch, N Eijkelkamp, RM Dijkhuizen, J Kesecioglu and CJ Heijnen. (2018). Intranasal stem cell treatment as a novel therapy for subarachnoid hemorrhage. Stem Cells Dev 27:313-325.

17. Liu W, R Li, J Yin, S Guo, Y Chen, H Fan, G Li, Z Li, X Li, et al. (2019). Mesenchymal stem cells alleviate the early brain injury of subarachnoid hemorrhage partly by 
suppression of Notch1-dependent neuroinflammation: involvement of Botch. J Neuroinflammation 16:8.

18. Scheller J, A Chalaris, D Schmidt-Arras and S Rose-John. (2011). The pro- and anti-inflammatory properties of the cytokine interleukin-6. Biochim Biophys Acta 1813:878-888.

19. Wang Z, Y Wang, X Tian, H Shen, Y Dou, H Li and G Chen. (2016). Transient receptor potential channel 1/4 reduces subarachnoid hemorrhage-induced early brain injury in rats via calcineurin-mediated NMDAR and NFAT dephosphorylation. Sci Rep 6:33577.

20. Uwe-Karsten H and K Helmut. (2007). Microglia: active sensor and versatile effector cells in the normal and pathologic brain. Nat Neurosci 10:1387-1394.

21. Garden GA and T Möller. (2006). Microglia biology in health and disease. J Neuroimmune Pharmacol 1:127.

22. Karim A, C Dominique, V Jacopo, D Annie, F Isabelle and S Michel. (2013). Involvement of nitric oxide through endocannabinoids release in microglia activation during the course of CNS regeneration in the medicinal leech. Glia 61: 636-649.

23. Pang J, J Peng, N Matei, P Yang, L Kuai, Y Wu, L Chen, MP Vitek, F Li, et al. (2018). Apolipoprotein E exerts a whole-brain protective property by promoting M1? Microglia quiescence after experimental subarachnoid hemorrhage in mice. Transl Stroke Res 9:654-668.

24. Qin C, WH Fan, Q Liu, K Shang, M Murugan, LJ Wu, W Wang and DS Tian. (2017). Fingolimod protects against ischemic white matter damage by modulating microglia toward M2 polarization via STAT3 pathway. Stroke 48: 3336-3346.

25. Hu X, P Li, Y Guo, H Wang, RK Leak, S Chen, Y Gao and J Chen. (2012). Microglia/macrophage polarization dynamics reveal novel mechanism of injury expansion after focal cerebral ischemia. Stroke 43:3063-3070.

26. Li R, W Liu, J Yin, Y Chen, S Guo, H Fan, X Li, X Zhang, $\mathrm{X}$ He and C Duan. (2018). TSG-6 attenuates inflammationinduced brain injury via modulation of microglial polarization in SAH rats through the SOCS3/STAT3 pathway. J Neuroinflammation 15:231.

27. Atangana E, UC Schneider, K Blecharz, S Magrini, J Wagner, M Nieminen-Kelha, I Kremenetskaia, FL Heppner, B Engelhardt and P Vajkoczy. (2017). Intravascular inflammation triggers intracerebral activated microglia and contributes to secondary brain injury after experimental subarachnoid hemorrhage (eSAH). Transl Stroke Res 8: 144-156.

28. Peng J, J Pang, L Huang, B Enkhjargal, T Zhang, J Mo, P $\mathrm{Wu}, \mathrm{W} \mathrm{Xu}, \mathrm{Y} \mathrm{Zuo}$, et al. (2019). LRP1 activation attenuates white matter injury by modulating microglial polarization through Shc1/PI3K/Akt pathway after subarachnoid hemorrhage in rats. Redox Biol 21:101121.

29. Peng S L. (2008). Foxo in the immune system. Oncogene 27:2337-2344.

30. Lundell LS, J Massart, A Altintas, A Krook and JR Zierath. (2019). Regulation of glucose uptake and inflammation markers by FOXO1 and FOXO3 in skeletal muscle. Mol Metab 20:79-88.

31. Cianciulli A, R Calvello, C Porro, T Trotta, R Salvatore and MA Panaro. (2016). PI3k/Akt signalling pathway plays a crucial role in the anti-inflammatory effects of curcumin in LPS-activated microglia. Int Immunopharmacol 36:282290.

32. Bradley J R. (2008). TNF-mediated inflammatory disease. J Pathol 214:149-160.
33. Stolfa DA, O Einsle, W Sippl and M Jung. (2012). Current trends in epigenetic drug discovery. Future Med Chem 4: 2029-2037.

34. Federico A, AJM Barbosa, P Marco Daniele and DR Alberto. (2013). Modulation of epigenetic targets for anticancer therapy: clinicopathological relevance, structural data and drug discovery perspectives. Curr Pharm Des 19: 578-613.

35. Ke Y, Z Run, S Chengmei, C Lei, L Peng, L Yi, P Lingmei, S Haitao, Q Kun and C Fanfan. (2013). Bone marrowderived mesenchymal stem cells maintain the resting phenotype of microglia and inhibit microglial activation. PLoS One 8:e84116.

36. Jaimes Y, Y Naaldijk, K Wenk, C Leovsky and F Emmrich. (2017). Mesenchymal stem cell-derived microvesicles modulate lipopolysaccharides-induced inflammatory responses to microglia cells. Stem Cells 35:812.

37. Lee JY, RF Keep, Y He, O Sagher, Y Hua and G Xi. (2010). Hemoglobin and iron handling in brain after subarachnoid hemorrhage and the effect of deferoxamine on early brain injury. J Cereb Blood Flow Metab 30:17931803.

38. Wang Z, B Yuan, F Fu, S Huang and Z Yang. (2017). Hemoglobin enhances miRNA-144 expression and autophagic activation mediated inflammation of microglia via mTOR pathway. Sci Rep 7:11861.

39. Burton MD, NL Sparkman and RW Johnson. (2011). Inhibition of interleukin- 6 trans-signaling in the brain facilitates recovery from lipopolysaccharide-induced sickness behavior. J Neuroinflammation 8:54.

40. Burchfield JS, Q Li, HY Wang and RF Wang. (2015). JMJD3 as an epigenetic regulator in development and disease. Int J Biochem Cell Biol 67:148-157.

41. Salminen A, K Kaarniranta, M Hiltunen and A Kauppinen. (2014). Histone demethylase Jumonji D3 (JMJD3/ KDM6B) at the nexus of epigenetic regulation of inflammation and the aging process. J Mol Med (Berl) 92:10351043.

42. Pereira GDC, GN Guimarães, AC Planello, MP Santamaria, APD Souza, SR Line and MR Marques. (2013). Porphyromonas gingivalis LPS stimulation downregulates DNMT1, DNMT3a, and JMJD3 gene expression levels in human HaCaT keratinocytes. Clin Oral Investig 17:12791285.

43. Tang Y, T Li, J Li, J Yang, H Liu, XJ Zhang and W Le. (2014). Jmjd3 is essential for the epigenetic modulation of microglia phenotypes in the immune pathogenesis of Parkinson's disease. Cell Death Differ 21:369-380.

Address correspondence to: Yong Jiang, PhD

Department of Neurosurgery The Affiliated Hospital of Southwest Medical University No. 25 Taiping Street Luzhou 646000 Sichuan

China

E-mail: jiangyong@swmu.edu.cn

Received for publication September 18, 2019

Accepted after revision December 23, 2019

Prepublished on Liebert Instant Online January 9, 2020 